\title{
High-Frequency QPOs and Overstable Oscillations of Black-Hole Accretion Disks
}

\author{
Dong Lai ${ }^{1}$, Wen Fu ${ }^{1,2}$, David Tsang ${ }^{1,3}$, Jiri Horak ${ }^{4}$ and Cong $\mathbf{Y u}^{1,5}$ \\ ${ }^{1}$ Department of Astronomy, Cornell University, Ithaca, NY 14853, USA \\ email: dong@astro.cornell.edu \\ ${ }^{2}$ Department of Physics \& Astronomy, Rice University, Houston, TX, USA \\ ${ }^{3}$ Theoretical Astrophysics, Caltech, Pasadena, CA, USA \\ ${ }^{4}$ Astronomical Institute of the Academy of Sciences, Prague, CZ \\ ${ }^{5}$ Yunnan Astronomical Observatory, Chinese Academy of Sciences, PRC
}

\begin{abstract}
The physical origin of high-frequency QPOs (HFQPOs) in black-hole X-ray binaries remains an enigma despite many years of detailed observational studies. Although there exists a number of models for HFQPOs, many of these are simply "notions" or "concepts" without actual calculation derived from fluid or disk physics. Future progress requires a combination of numerical simulations and semi-analytic studies to extract physical insights. We review recent works on global oscillation modes in black-hole accretion disks, and explain how, with the help of general relativistic effects, the energy stored in the disk differential rotation can be pumped into global spiral density modes in the disk, making these modes grow to large amplitudes under certain conditions ("corotational instability"). These modes are robust in the presence of disk magnetic fields and turbulence. The computed oscillation mode frequencies are largely consistent with the observed values for HFQPOs in BH X-ray binaries. The approximate 2:3 frequency ratio is also expected from this model. The connection of HFQPOs with other disk properties (such as production of episodic jets) is also discussed.
\end{abstract}

Keywords. accretion disks - hydrodynamics - MHD - black hole physics - X-ray: binaries

\section{Introduction}

High-Frequency Quasi-Periodic Oscillations (HFQPOs) in the X-ray fluxes have been observed from a number of black-hole (BH) X-ray binaries since the late 1990s. Their frequencies $(40-450 \mathrm{~Hz})$ are comparable to the orbital frequencies at the Innermost Stable Circular Orbit (ISCO) of BHs with mass $M \sim 10 M_{\odot}$, and do not vary significantly in response to sizeable (factors of 3-5) changes in the luminosity. Compared to the lowfrequency QPOs, the HFQPOs have low amplitudes $(0.5-2 \% \mathrm{rms}$ at $2-60 \mathrm{keV})$ and low coherence $(Q \sim 2-10)$, and are only observed in the "intermediate state" (or "steep power law state") of the X-ray binaries (for reviews, see Remillard \& McClintock 2006; Belloni et al. 2012). In addition, several systems show pairs of QPOs with frequency ratios close to $2: 3$. It is generally recognized that HFQPOs may provide important information about the BH (mass and spin) and about the dynamics of inner-most accretion flows.

Current status of QPO Theory/Models: The physical origin of HFQPOs is currently unclear. Below we comment on some of the ideas/models have been suggested.

(i) In the spot models (Stella et al. 1999), the HFQPOs arise from the Doppler modulation of the radiation from spots orbiting in the inner part of the $\mathrm{BH}$ accretion disk. It is not clear how the position of the spot (a free parameter in these models) is determined and how the spot can survive the differential rotation of the disk.

(ii) Another class of models identify HFQPOs with various oscillation modes of a finite, pressure-supported accretion torus (Rezzolla et al. 2003; Blaes et al. 2006). It is not clear 
that the accretion flow can be approximated by a torus and how the position and size of the torus are determined.

(iii) The harmonic relation between the observed frequencies led Abramowicz \& Kluzniak (2001) to suggest that the HFQPOs are a result of a nonlinear resonance. However, so far no fluid dynamical model producing these resonances has been developed. Detailed calculations of the resonant coupling between the epicyclic modes in slender tori indicates that such resonance is very weak (Horák 2008).

(iv) A large class of theoretical models is based on the relativistic diskoseismology (Kato 2001). The $g$-modes (also called inertial-gravity modes) have attracted most attention because their existence does not require a reflective inner disk boundary. These oscillations have at least one node in the vertical direction and their restoring force results from rotation and gravity. Unfortunately, the non-axisymmetric $g$-modes are either damped due to corotation resonance (Kato 2003; Li et al. 2003) or have frequencies too high compared to HFQPOs. The axisymmetric $g$-mode trapped around the maximum of the radial epicyclic frequency may account for some single HFQPO frequencies (see Wagoner 2012), and several studies suggested that they may be resonantly excited in the warped or eccentric disks (Kato 2008; Ferreira \& Ogilvie 2008). However, the self-trapping property of $g$-modes can be easily destroyed by a weak (sub-thermal) disk magnetic field (Fu \& Lai 2009) and turbulence (Arras et al. 2006; Reynolds \& Miller 2009).

Related to (iv) above, perhaps more promising is the disk $p$-modes. In the following, we review recent works on disk $p$-modes from the Cornell group and other related efforts (see Lai \& Tsang 2009, Tsang \& Lai 2008,2009a,b,c; Fu \& Lai 2009,2011a,b,2012; Horak \& Lai 2012).

\section{Disk P-modes: Corotational Instability}

Disk $p$-modes (also called inertial acoustic modes) represent nearly horizontal oscillations with almost no vertical structure. They are trapped between the inner boundary of the disk and the inner Lindblad resonance (ILR; see below) Because of their simple $2 \mathrm{D}$ structure, the basic wave properties of the p-modes (e.g., propagation diagram) are not strongly affected by disk magnetic fields (Fu \& Lai 2009) and are likely robust in the presence of disk turbulence. Lai \& Tsang (2009) (see also Tsang \& Lai 2008,2009c) showed that the non-axisymmetric $p$-modes can naturally grow due to the corotational instability. This can be understood as follows.

Consider a 2D fluid disks without self-gravity and magnetic field. For the disturbances of the form $e^{i m \phi-i \omega t}$, where $m>0$ and $\omega$ is the wave (angular) frequency, the WKB dispersion relation for density waves reads

$$
\tilde{\omega}^{2}=\kappa^{2}+k_{r}^{2} c_{s}^{2}, \quad(\text { where } \tilde{\omega} \equiv \omega-m \Omega)
$$

where $\Omega(r)$ is the disk rotation frequency, $\kappa(r)$ is the radial epicyclic frequency, $k_{r}$ is the radial wavenumber, and $c_{s}$ is the sound speed. Thus waves can propagate either inside the Inner Lindblad resonance (ILR, defined by $\tilde{\omega}=-\kappa$ ) or outside the Outer Lindblad resonance (OLR, $\tilde{\omega}=\kappa)$. The region between ILR and OLR is evanescent ("corotation barrier") except for a narrow "Rossby zone" near the corotation resonance (CR, where $\tilde{\omega}=0$ ). The significance of CR is that waves can be absorbed by the background flow since the wave patten speed $\omega / m$ matches the flow rotation rate $\Omega$ at CR. Depending on the sign of $d \zeta / d r$, where $\zeta \equiv \kappa^{2} /(2 \Omega \Sigma)$ is the vortensity of the background flow ( $\Sigma$ is the surface density of the disk), the Rossby zone can lie just inside or outside CR, and the corotational wave absorption can be either positive or negative (see Fig. 1). 
This corotational instability of non-axisymmetric $p$-modes arises because of two effects: (1) Since the waves inside the ILR carry negative energies while those outside the OLR carry positive energies, the leakage of the p-waves through the corotation barrier (between ILR and OLR) leads to mode growth. (2) More importantly, when the vortensity of the disk flow, $\zeta=\kappa^{2} /(2 \Omega \Sigma)$ [this applies to barotropic fluid, for which the pressure is a unique function of the density; see Tsang \& Lai (2009c) for the non-barotropic case], has a positive slope at the corotation radius wave absorption at the corotation resonance leads to amplification of the trapped p-mode.

General Relativity plays an important role in the corotational instability of p-modes. In Newtonian theory, $\kappa=\Omega$, so $d \zeta / d r<0$ when $\Sigma$ does not vary strongly and hence the mode cannot grow. In GR, $\kappa$ reaches a maximum at a finite radius before dropping to zero at $r_{\mathrm{ISCO}}$. Thus, if the mode frequency is such that $d \zeta / d r>0$ at corotation, then the mode can grow.

\section{Overstable P-modes and HFQPOs}

Our linear calculations and nonlinear simulations (based on pseudo-Newtonian potential) have demonstrated the corotational instability of disk p-modes (Lai \& Tsang 2009; Tsang \& Lai 2009; Fu \& Lai 2011a,2012). In particular, the low-order p-modes (trapped between the inner disk edge and the ILR) have the following properties: (i) The mode frequency is $\omega \simeq \beta m \Omega_{\text {in }}$, where $\beta=0.5-0.75$ (depending on the disk models and the inner boundary condition. The mode frequency varies (by about 10\%) as $\dot{M}$ changes by a factor of 3 . The frequency is consistent with the estimated $\mathrm{BH}$ mass and spin of X-ray binaries. The frequency ratio is approximately $1: 2: 3, \cdots$, but not exactly. (ii) The mode growth rate (due to corotation resonance) is much smaller for $m=1$ than for $m=2,3,4, \cdots$. Since high- $m$ modes are hard to observe, it is reasonable to expect that
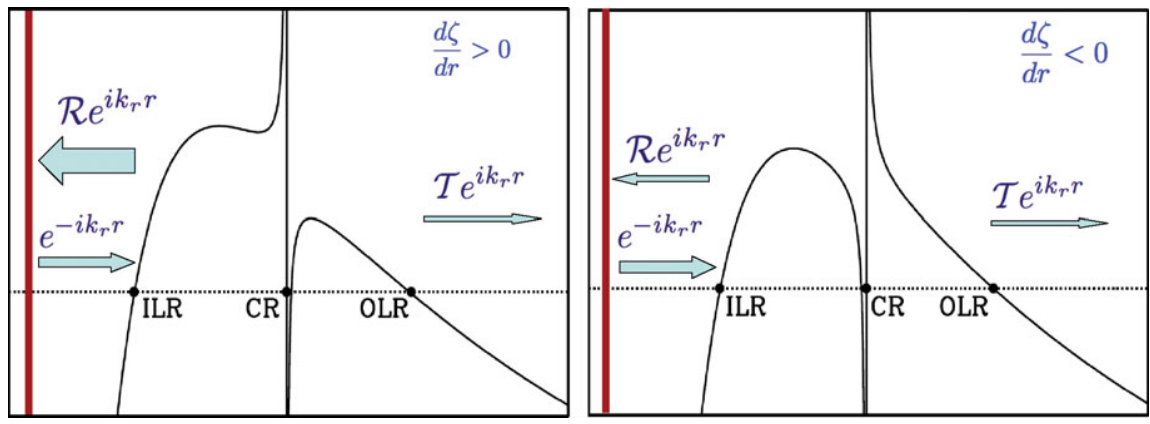

Figure 1. Super-reflection and corotational instability of nonaxisymmetric waves in rotating disks. The solid curves depict the effective potential for wave propagation: Density waves can propagate either inside the ILR or outside the OLR. Between the ILR and OLR lies the corotation resonance $(\mathrm{CR})$ and the associated Rossby zone (where the effective potential is negative). An incident wave $e^{-i k_{r} r}$ propagating from small radii towards the corotation barrier will be partially reflected $\left(\mathcal{R} e^{i k_{r} r}\right)$ and partially transmitted $\left(\mathcal{T} e^{i k_{r} r}\right)$. Since the waves inside CR $\left(r<r_{\mathrm{CR}}\right)$ carry negative energies, while those outside CR carry positive energies, we have $(-1)=(-1)|\mathcal{R}|^{2}+|\mathcal{T}|^{2}+\mathcal{D}_{c}$ from energy conservation, or $|\mathcal{R}|^{2}=1+|\mathcal{T}|^{2}+\mathcal{D}_{c}$, where $\mathcal{D}_{c}$ is the wave energy absorption at CR. When the background disk has $d \zeta / d r>0$ at CR [where $\zeta=\kappa^{2} /(2 \Omega \Sigma)$ is the vortensity], the Rossby zone lies just outside CR, thus $\mathcal{D}_{c}>0$ and $|\mathcal{R}|^{2} \simeq 1+\mathcal{D}_{c}>1$, we have super-reflection (the left panel). If the disk has a reflective inner boundary (represented by the red vertical line), then the stationary waves (modes) trapped between $r_{\text {in }}$ and ILR will be overstable (with growing amplitude). When the background disk has $d \zeta / d r<0$, there is no super-reflection $(|\mathcal{R}|<1)$ and the modes are damped by the corotational wave absorption (the right panel). 
the most visible modes are $m=2,3$. (iii) The nonlinear mode frequency is close to the linear mode frequency ( $\mathrm{Fu} \&$ Lai 2012, in prep).

All the above make p-modes a promising candidate for explaining HFQPOs in $\mathrm{X}$ ray binaries. However, there are a number of issues and complications that must be considered:

(i) Mode Damping due to Radial Infall: For standard thin disks, the radial infall velocity increases rapidly near $r_{\mathrm{ISCO}}$ - this can be described in the slim disk model. Such infall tends to damp the p-modes (but not completely, because of the sharp density gradient around $r_{\text {ISCO }}$; see Lai \& Tsang 2009). So we have a competition between mode growth (due to corotation resonance) and damping (due to infall). The net result is that for standard thin disks, p-modes are likely damped. This is consistent with the observations that no HFQPOS are detected in the thermal (low-soft) state of BH X-ray binaries.

(ii) Effects of magnetic fields: However, real disks (particularly those in the "intermediate state" when HFQPOs are observed) are more complicated, and have magnetic fields. As noted above, the p-mode frequencies are only slightly affected by the magnetic field, provided that $r_{\text {in }}$ is close to $r_{\mathrm{ISCO}}$. However, the mode growth rates can be significant modified by the $B$-field. There are three effects: (1) Toroidal fields inside the disk tend to surpress the corotational instability (Fu \& Lai 2011a,b). (2) Magnetic fields can accumulate inside $r_{\mathrm{ISCO}}$, forming a magnetosphere around the $\mathrm{BH}$. Thus, the inner disk edge may be more reflective than the standard thin disk (Tsang \& Lai 2009b; Fu \& Lai 2012). This can enhance the net growth rate of p-modes. (3) Large-scale poloidal magnetic fields threading the disk can provide a coupling between the disk and the disk magnetosphere (Yu \& Lai 2013, in prep). This tends to enhance the corotational instability and increase the growth rate of p-modes (see also Tagger \& Varniere 2006).

The last two points may be of particular importance. Large-scale magnetic fields provide a natural means to launch jets/outflows from accretion disks. Magnetic fields threading the BH can also lead to jets from the rotating BHs (McKinney et al. 2012). In BH $\mathrm{x}$-ray binaries, episodic (ballistic) jets are observed in the intermediate state, and this is the same state during which HFQPOs are detected. In our model, the disk and corona (coupled by a large-scale poloidal $B$-field) oscillate together, with the "clock" mainly set by the disk dynamics. The corona is important, however, since it can lead to the variation of hard x-ray photon flux as observed in HFQPOs.

The current understanding of accretion flows in the intermediate state is rather poor (Done et al. 2007). It is even not clear that $r_{\text {in }}$ extends to $r_{\mathrm{ISCO}}$ in this state. We suggest that in the intermediate state, large-scale $B$-fields are temporarily/episodically created (e.g., magnetic fields buoyantly rise from the collapsed ADAF state and followed by reconnection). This will then allow the p-modes to grow and manifest as HFQPOs; at the same time, it will lead to the production of episodic jets.

(iii) Full GR Calculations. Horak \& Lai (2012) presented full GR calculations of overstable p-modes of $\mathrm{BH}$ accretion disks. In particular, they derived the GR version of the corotational instability criterion and generalized the Newtonian disk vortensity. The results are qualitatively in agreement with the pseudo-Newtonian results discussed above, with one important difference: For Kerr BHs with the spin parameter close to unity, the mode frequency is close to $m \Omega_{\text {in }}$ (i.e., $\beta \simeq 1$ ). Assuming $r_{\text {in }}=r_{\text {ISCO }}$, this would lead to a strong tension between the simplest p-mode theory and the observational constraints on the BH mass and spin. The discrepency becomes most severe for GRS 1915+105 $\left(M=14 \pm 4.4 M_{\odot}\right)$, which may have two pairs of HFQPOs (41 and $67 \mathrm{~Hz}, 113$ and $168 \mathrm{~Hz}$ ), and whose spin parameter has been constrained to be $a>0.975 \mathrm{using}$ the continuum fitting method (see Narayan \& McClintock 2012). 
Overall, it appears that for the simplest disk models, non-axisymmetric p-modes have frequencies that are too high compared to the observation of HFQPOs in $\mathrm{BH}$ X-ray binaries, at least for some systems. Several effects may decrease the theoretical p-mode frequencies. For example, a higher disk sound speed leads to lower mode frequencies, and a steeper surface density profile (larger $p$ in $\Sigma \propto r^{-p}$ ) reduces $\omega_{\min }$, the minimum frequency for the corotational wave absorption to amplify the mode. Magnetic fields may also play an important role [see (ii) above]. In particular, our calculations so far assume that the inner disk radius coincides with the ISCO. This may not be the case during the "intermediate state" (Done et al. 2007; Oda et al. 2010). As noted before, a major uncertainty in calculating the disk p-modes is the inner disk boundary condition. When magnetic fields advect inwards in the accretion disk and accumulate around the $\mathrm{BH}$, (Bisnovatyi-Kogan \& Ruzmaikin 1974; Igumenshchev et al. 2003; Rothstein \& Lovelace 2008 ), the inner disk radius $r_{\text {in }}$ may be larger than $r_{\text {ISCO }}$. This reduces $\Omega_{\text {in }}$ relative to $\Omega_{\text {ISCO }}$, leading to lower p-mode frequencies.

Acknowledgements: This work has been supported in part by NSF grants AST1008245 and AST-1211061, and NASA grants NNX12AF85G and NNX10AP19G.

\section{References}

Abramowicz, M. A. \& Kluzniak, W. 2001, A\&SA, 374, L19

Arras, P., Blaes, O. M., \& Turner, N. J. 2006, ApJ, 645, L65

Belloni, T. M., Sanna, A., \& Méndez, M. 2012, MNRAS, in press (axrXiv:1207.2311)

Bisnovatyi-Kogan, G. S. \& Ruzmaikin, A. A. 1974, Ap\&SSS, 28, 45

Done, C., Gierlinski, M., \& Kubota, A. 2007, Astron. Astrophys. Rev., 15, 1

Ferreira, B. T. \& Ogilvie, G. I. 2008, MNRAS, 386, 2297

Fu, W. \& Lai, D. 2009, ApJ, 690, 1386

Fu, W. \& Lai, D. 2011a, MNRAS, 410, 399

Fu, W. \& Lai, D. 2011b, MNRAS, 410, 1617

Fu, W. \& Lai, D. 2012, MNRAS, 423, 831

Horák, J. 2008, A\& A, 486, 1

Horák, J. \& Lai, D. 2012, MNRAS, submitted

Igumenshchev, I. V., Narayan, R., \& Abramowicz, M. A. 2003, ApJ, 592, 1042

Kato, S. 2001, PASJ, 53, 1

Kato, S. 2003, PASJ, 55, 257

Kato, S. 2008, PASJ, 60, 111

Lai, D., Tsang D. 2009, MNRAS, 393, 979

Li, L., Goodman, J., \& Narayan, R. 2003, ApJ, 593, 980

McKinney, J. C., Tchekovskoy, A., \& Blandford, R. D. 2012, MNRAS, 423, 3083

Meheut, H., Yu, C., \& Lai, D. 2012, MNRAS, 422, 2399

Narayan, R., Goldreich, P., \& Goodman, J. 1987, MNRAS, 228, 1

Narayan, R. \& McClintock, J. 2012, MNRAS, 419, L69

Oda H., et al. 2010, ApJ, 712, 639

Remillard, R. A. \& McClintock, J. E. 2006, ARA\&A, 44, 49

Reynolds, C. S. \& Miller, M. C. 2009, ApJ, 692, 869

Rothstein, D. M. \& Lovelace, R. V. E.. 2008, ApJ, 677, 1221

Stella, L., Vietri, M., \& Morsink, S. M. 1999, ApJ, 524, L63

Tagger, M. \& Varniere, P. 2006, ApJ, 652, 1457

Tsang, D. \& Lai, D. 2008, MNRAS, 387, 446

Tsang, D. \& Lai, D. 2009a, MNRAS, 393, 992

Tsang, D. \& Lai. D. 2009b, MNRAS, 396, 589

Tsang, D. \& Lai, D. 2009c, MNRAS, 400, 470

Wagoner, R. V. 2012, ApJ, 752, L18 